1 Hacettepe Journal of Mathematics and Statistics

$\bigcap$ Volume 45 (5) (2016), 1393-1410

\title{
Numerical solution of linear integro-differential equation by using modified Haar wavelets
}

\author{
Fernane Khaireddine ${ }^{* \dagger}$ and Ellaggoune Fateh ${ }^{\ddagger}$
}

\begin{abstract}
In this paper, we introduce a numerical method for solving linear Fredholm integro-differential equations of the first order. To solve these equations, we consider the equation solution approximately from rationalized Haar $(R H)$ functions.

The numerical solution of a linear integro-differential equation reduces to solving a linear system of algebraic equations. Also, Some numerical examples are presented to illustrate the efficiency of the method.
\end{abstract}

Keywords: Block-Pulse Functions, Operational matrix, Volterra integral equations, Integro-differential equations, RHFs.

2000 AMS Classification: 65R20, 33D45, 45D05, 45F05

Received: 12.04.2015 Accepted: 13.02.2016 Doi: 10.15672/HJMS.20164515687

\section{Introduction}

Some important problems in science and engineering can usually be reduced to a system of integral and integro-differential equations. Integro-differential equations have attracted much attention and solving such equations has been one of the interesting tasks for mathematicians. Several methods have been proposed for numerical solution of these equations (see, e.g., [12]). One technique is the collocation method; of numerous research papers about this approach we cite here ([6], [18]). Since 1991 the wavelet method has been applied to solving integral equations. Various wavelet bases have been employed. In addition to the conventional Daubechies wavelets [12], the Hermite-type trigonometric wavelets [8], linear B-splines [2], Walsh functions [9], Cohen [8] and Fariborzi [10] wavelets have been used. These solutions are often quite complicated, therefore simplifications are welcome. One possibility is to make use of Haar wavelets, which are mathematically the simplest wavelets. For linear integral equations this approach has

*Department of Mathematics, University of 8 May 1945 Guelma, Algeria, Email: kfernane@yahoo.fr

${ }^{\dagger}$ Fernane Khaireddine.

${ }^{\ddagger}$ Department of Mathematics, University of 8 May 1945 Guelma, Algeria, Email: fellaggoune@gmail.com 
been realized in ([5], [13]). In this paper we examine the rate of convergence of the modified rationalized method using Haar functions for solving Fredholm integro-Differential equations combined with finite difference methods.

Solving the algebraic system obtained by the $(R H)$ functions method allows one to obtain first derivative approximations using a central difference scheme. We apply the proposed method on some test problems to show its accuracy and efficiency. Also, the error evaluation of this method is presented. Before starting, let us recall some definitions.

1.1. Definition. ([5]) The Haar wavelet is the function defined on the real line $\mathbb{R}$ as:

$$
H(t)=\left\{\begin{array}{cc}
1, & 0 \leq t<\frac{1}{2} \\
-1, & \frac{1}{2} \leq t<1 \\
0, & \text { otherwise }
\end{array}\right.
$$

The Haar wavelet $H(t)$ can be used to define a sequence of one-dimensional $(R H)$ functions on $[0,1)$ as follows:

1.2. Definition. ([5]) The $(R H)$ functions $h_{n}(t)$, for $n=2^{i}+j$ with $i \in \mathbb{Z}$ and $j=$ $0,1, \ldots, 2^{i-1}$, are the functions defined on the interval $[0,1)$ as:

$$
h_{n}(t)=H\left(2^{n} t-j\right)_{\mid[0,1)}
$$

Also, we define $h_{0}(t)=1$ for all $t \in[0,1)$.

In Eq.(1.2), are the orthogonal set of rationalized Haar functions and can be defined on the interval $[0,1)$ as [17]:

$$
R H(r, t)=h_{r}(t)=\left\{\begin{aligned}
1, & \text { if } J_{1} \leq t<J_{\left(\frac{1}{2}\right)} \\
-1, & \text { if } J_{\left(\frac{1}{2}\right)} \leq t<J_{0} \\
0, & \text { otherwise }
\end{aligned}\right.
$$

where, $J_{u}=\frac{j-u}{2^{i}}, u=0, \frac{1}{2}, 1$.

The value of $r$ is defined by two parameters $i$ and $j$ as:

$$
r=2^{i}+j-1, i=0,1,2 \ldots, j=1,2, \ldots, 2^{i}
$$

$h_{0}(t)$ is defined for $i=j=0$ and given by:

$$
h_{0}(t)=1,0 \leq t<1
$$

$h_{0}(t)$ is also included to make this set complete. The orthogonality property is given by:

$$
\int_{0}^{1} R H(r, t) R H(v, t) d t= \begin{cases}2^{-i}, & r=v \\ 0 & r \neq v\end{cases}
$$

where

$$
v=2^{n}+m-1, n=0,1,2,3, \ldots, m=1,2,3, \ldots, 2^{n}
$$




\section{Function Approximation}

Any function $f(t)$ defined over the interval $[0,1)$, which is $\mathbb{L}^{2}([0,1))$, can be expanded in $(R H)$ functions as $([23])$;

$$
f(t)=\sum_{r=0}^{+\infty} \alpha_{r} R H(r, t), r=0,1,2, \ldots .
$$

where the $(R H)$ function coefficients $\alpha_{r}$ are given by:

$$
\alpha_{r}=\frac{\langle f(t), R H(r, t)\rangle}{\langle R H(r, t), R H(r, t)\rangle}=2^{i} \int_{0}^{1} f(t) R H(r, t) d t, r=0,1,2, \ldots
$$

with $r=2^{j}+i-1, i=0,1,2,3, \ldots, j=1,2,3, \ldots, 2^{n}$ and $r=0$ for $i=j=0$.

Usually, the series expansion of Eq. (2.1) contains infinite terms. If $f(t)$ is piecewise constant by itself, or may be approximated as piecewise constant during each subinterval, then Eq. (2.1) will be terminated at finite terms. Otherwise, it is truncated up to its first $m$ terms as:

$$
f(t) \approx \sum_{r=0}^{k-1} a_{r} R H(r, t)=A^{T} \phi(t)
$$

where $k=2^{\alpha+1}$, and $\alpha=0,1,2,3, \ldots$

The $(R H)$ function coefficients vector $\phi(t)$ and $(R H)$ functions vector $h(t)$ are defined as;

$$
A=\left[a_{0}, a_{1}, a_{2}, \ldots, a_{k-1}\right]^{T}
$$

and

$$
\phi(t)=\left[h_{0}, h_{1}, h_{3}, \ldots . ., h_{k-1}\right]^{T}
$$

where

$$
h_{r}(t)=R H(r, t), r=0,1,2, \ldots, k-1
$$

Babolian et al. proved in [21] that:

$$
\begin{aligned}
& \left\|f(t)-\sum_{r=0}^{k-1} a_{r} R H(r, t)\right\|_{\mathbb{L}^{2}}^{2}==\left\|\sum_{r=k}^{+\infty} a_{r} h_{r}(t)\right\|_{\mathbb{L}^{2}}^{2} \\
& \leq \sum_{r=k}^{+\infty}\left|a_{r}\right|^{2}\left\|h_{r}(t)\right\|_{\mathbb{L}^{2}}^{2} \\
& =\sum_{r=k}^{+\infty}\left|a_{r}\right|^{2} \\
& \sim \sum_{r=k}^{+\infty} 2^{-r} \sim 2^{-k}=O\left(2^{-k}\right) \leq C 2^{-k}
\end{aligned}
$$


where $C$ is a constant of integration.

Now, let $k(t, s)$ be a function of two independent variables defined for $t \in[0,1)$ and $s \in[0,1)$. Then $k(t, s)$ can be expanded in $(R H)$ functions as:

$$
k(t, s)=\sum_{u=0}^{m-1} \sum_{v=0}^{m-1} h_{u v} h_{v}(t) h_{u}(s)
$$

In Eq. (2.8) $h_{u v}$, for $u=0,1,2, . ., m-1$ and $v=0,1,2, \ldots, m-1$, is given as:

$$
h_{u v}=2^{i+q} \int_{0}^{1} \int_{0}^{1} k(s, t) h_{v}(t) h_{u}(s) d t d s
$$

where

$u=2^{i}+j, i \geq 0$ and $0 \leq j<2^{i} v=2^{q}+r, q \geq 0$ and $0 \leq r<2^{q}$ hence we have

$$
k(t, s)=\phi^{T}(t) H \phi(s)
$$

where

$$
H=\left(\hat{\Phi}_{k \times k}^{-1}\right)^{T} \hat{H} \hat{\Phi}_{k \times k}^{-1}
$$

with

$$
\hat{H}=\left(h_{u v}\right)_{k \times k}^{T}
$$

Where $\hat{H}$ is an $k \times k$ matrix such that:

$$
h_{i j}=\frac{\langle R H(i, t),\langle k(t, s), R H(j, s)\rangle\rangle}{\langle R H(i, t), R H(i, t)\rangle\langle R H(j, t), R H(j, t)\rangle}
$$

Take the Newton-Côtes nodes as:

$$
\begin{aligned}
& t_{i}=\frac{2 i-1}{2 k}, i=1,2, . ., k \\
& \hat{h}_{l p}=k\left(\frac{2 l-1}{2 k}, \frac{2 p-1}{2 k}\right), p, l=1,2, \ldots, k .
\end{aligned}
$$

2.1. Operational matrix of integration. Discrete Haar functions of order $k$ represented by $2^{k} \times 2^{k}$ matrix $\hat{\Phi}_{k \times k}$, in the sequency ordering are given by the following recurrence relation $([23])$ :

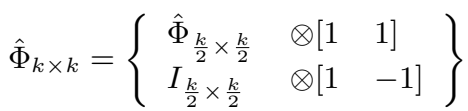

(2.17) $\Phi_{1 \times 1}=[1]$

where $I_{\frac{k}{2} \times \frac{k}{2}}$ is the identity matrix of dimension $k$ and $\otimes$ is the Kronecker product.

The integration of $(R H)$ functions can be expanded into Haar series with Haar coefficient matrix $P$ as follows: 


$$
\int_{0}^{1} t \phi(x) d x=P \phi(t)
$$

The $k \times k$ square matrix $P=P_{k}$ is called the operational matrix of integration and is given in [7] as:

$$
P_{k}=\frac{1}{2 k}\left(\begin{array}{cc}
2 k P_{\frac{k}{2}} & -\hat{\Phi}_{\frac{k}{2}} \\
-\hat{\Phi}_{\frac{k}{2}} & 0
\end{array}\right)
$$

where $\hat{\Phi}_{1}^{-1}=[1], P_{1}=\left[\frac{1}{2}\right], \hat{\Phi}_{k}$ is given by Eq. (2.16) and

$$
\hat{\Phi}_{k}^{-1}=\frac{1}{k} \hat{\Phi}_{k}^{T} \operatorname{diag}(1,1,2,2, \underbrace{2^{2}, . ., 2^{2}}_{2^{2}}, \ldots, \underbrace{2^{\alpha-1}, \ldots, 2^{\alpha-1}}_{2^{\alpha-1}})
$$

Also, the integration of the cross-product of two $(R H)$ function vector is:

$$
\int_{0}^{1} \phi(t) \phi^{T}(t) d t=D
$$

where $D$ is a diagonal matrix given by:

$$
D=\operatorname{diag}(1,1,2,2, \underbrace{2^{2}, \ldots, 2^{2}}_{2^{2}}, \ldots, \underbrace{2^{\alpha-1}, \ldots, 2^{\alpha-1}}_{2^{\alpha-1}})
$$

2.2. The product operational matrix. ([23])

Let the product of $\phi(t)$ and $\phi^{T}(t)$ be called the $(R H)$ product matrix $\psi_{k \times k}(t)$. That is:

(2.23) $\phi(t) \phi^{T}(t)=\psi_{k \times k}(t)$

The basic multiplication properties of $(R H)$ functions are as:

(2.24) $\quad h_{0}(t) h_{i}(t)=h_{i}(t), i=0,1, \ldots, m-1$

and for $i<j$, we have

$$
h_{i}(t) h_{j}(t)=\left\{\begin{array}{rr}
h_{j}(t), & \text { if } h_{j} \text { occurs during the positive half-wave of } h_{i} \\
-h_{j}(t), & \text { if } h_{j} \text { occurs during the negative half-wave of } h_{i} \\
0 & \text { otherwise. }
\end{array}\right.
$$

Also, the square of any $(R H)$ functions is a block-pulse, with magnitude unity during both the positive and negative half-waves of $(R H)$ functions.

For notation simplification, let us define:

$$
\begin{aligned}
& \hat{\phi}_{a}(t)=\left[h_{0}(t), \ldots, h_{k / 2-1}(t)\right]^{T} \\
& \hat{\phi}_{b}(t)=\left[h_{k / 2}(t), \ldots, h_{k-1}(t)\right]^{T}
\end{aligned}
$$


The matrix $\psi_{k \times k}(t)$ in Eq. (2.23) can be derived easily as follows from ([7]):

$$
\psi_{k \times k}(t)=\left[\begin{array}{ll}
\psi_{k / 2}(t) & D_{k / 2} \operatorname{diag}\left[\hat{\phi}_{b}(t)\right] \\
\operatorname{diag}\left[\hat{\phi}_{b}(t)\right] D_{k / 2}^{T} & \operatorname{di\alpha g}\left[D_{k / 2}^{-1} \hat{\phi}_{a}(t)\right]
\end{array}\right]
$$

where

$$
\text { (2.29) } \quad \psi_{1}(t)=\left[h_{0}(t)\right]
$$

With the above recursive formulas, we can evaluate $\psi_{k}(t)$ for any $k=2^{\alpha}$, where $\alpha$ is a positive integer. Furthermore, by multiplying the matrix $\psi_{k}(t)$ in Eq. $(2.23)$ by the vector $A$ in Eq. (2.3) we obtain:

$$
\psi_{k}(t) A=\tilde{A}_{k} \phi(t)
$$

Where $\tilde{A}_{k}$ is a $k \times k$ given by [7]:

$$
\tilde{A}_{k}=\left[\begin{array}{ll}
\tilde{A}_{k / 2}(t) & D_{k / 2} \operatorname{diag}\left[\tilde{c}_{b}\right] \\
\operatorname{diag}\left[\tilde{c}_{b}\right] D_{k / 2}^{-1}(t) & \operatorname{diag}\left[\tilde{c}_{b}^{T} D_{k / 2}\right]
\end{array}\right]
$$

where $C_{1}=c_{0}$, and

$$
\tilde{c}_{a}=\left[c_{0}, \ldots, c_{k / 2-1}\right]^{T}
$$

$$
\tilde{c}_{b}=\left[c_{k / 2}, \ldots, c_{k-1}\right]^{T}
$$

\section{Application of HAAR wavelet method}

3.1. Solution of the Linear Fredholm Integro-Differential Equation. Consider the linear Fredholm integro-differential equation given by:

$$
\left\{\begin{array}{l}
q(t) y^{\prime}(t)=\int_{0}^{1} k(t, s) y(s) d s+r(t) y(t)+x(t) \\
y(0)=y_{0}
\end{array}\right.
$$

where the functions $x, q, r \in \mathbb{L}^{2}([0,1))$, the kernel $k \in \mathbb{L}^{2}([0,1) \times[0,1))$ are known and $y(t)$ is the unknown function to be determined.

We approximate $x, q, r, y^{\prime}$ and $k$ using Haar wavelet space as follows:

$$
\left\{\begin{array}{l}
y(t)=Y^{T} \phi(t)=\phi^{T}(t) Y \\
y^{\prime}(t)=Y^{\prime T} \phi(t)=\phi^{T}(t) Y^{\prime} \\
y(0)=Y_{0}^{T} \phi(t)=\phi^{T}(t) Y_{0} \\
x(t)=X^{T} \phi(t)=\phi^{T}(t) X \\
k(t, s)=\psi^{T}(t) K \psi(s)=\psi^{T}(s) K^{T} \psi(t) \\
r(t)=R^{T} \phi(t)=\phi^{T}(t) R \\
q(t)=Q^{T} \phi(t)=\phi^{T}(t) Q
\end{array}\right.
$$

where $\phi(t)$ is given by Eq. (2.5) and $Y$ is an unknown $m \times 1$ vector.

$k$ is a known $m \times m$ dimensional matrix given by Eq. (2.8) and $X$ is a known $m \times 1$ vector given by Eq. (2.3). 
Substituting Eq. (3.2) into (3.1) we have:

$$
\begin{aligned}
Q^{T} \phi(t) \phi^{T}(t) Y^{\prime}= & \int_{0}^{t} \phi^{T}(t) H \phi(s) \phi^{T}(s)\left(P^{T} Y^{\prime}+Y_{0}\right) d s \\
& +R^{T} \phi(t) \phi^{T}(t)\left(P^{T} Y^{\prime}+Y_{0}\right)+X^{T} \phi(t)
\end{aligned}
$$

we have $\phi(t) \phi^{T}(t)=\psi_{k \times k}(t)$

$$
\begin{aligned}
Q^{T} \psi_{k \times k}(t) Y^{\prime}= & \int_{0}^{t} \phi^{T}(t) H \psi_{k \times k}(s)\left(P^{T} Y^{\prime}+Y_{0}\right) d s \\
& +R^{T} \psi_{k \times k}(t)\left(P^{T} Y^{\prime}+Y_{0}\right)+X^{T} \phi(t) \\
Q^{T} \psi_{k \times k}(t) Y^{\prime}= & \phi^{T}(t) H \int_{0}^{t} \psi_{k \times k}(s)\left(P^{T} Y^{\prime}+Y_{0}\right) d s \\
& +R^{T} \psi_{k \times k}(t)\left(P^{T} Y^{\prime}+Y_{0}\right)+X^{T} \phi(t)
\end{aligned}
$$

by Eq. (2.21) and by Eq. (2.23), we have $Q^{T} \psi_{k \times k}(t)=\psi_{k \times k}(t) Q=\widetilde{Q} \phi(t)$

$$
\phi^{T} \widetilde{Q} Y^{\prime}=\phi^{T}(t) H D\left(P^{T} Y^{\prime}+Y_{0}\right)+\phi^{T}(t) \widetilde{R}\left(P^{T} Y^{\prime}+Y_{0}\right)+\phi^{T}(t) X
$$

or

$$
\left(\widetilde{Q}-H D P^{T}-R P^{T}\right) Y^{\prime}=H D P Y_{0}+\widetilde{R} Y_{0}+X
$$

By solving this linear system we can obtain the vector $Y^{\prime}$. Thus,

$$
y^{\prime}(t)=Y^{\prime T} \phi(t)=\phi^{T}(t) Y^{\prime}
$$

Eq. (3.9) can be solved for the unknown vector $Y^{\prime}$.

The numerical solution $y_{k}$ is obtained by using finite differences formulas to approximate the first time derivative. In general, the first order derivative of second order error central difference formula can be derived from the Taylor series expansion as follows:

\section{The Algorithm}

\section{Step 1:}

Put $h=\frac{1}{k}, k \in \mathbb{N},, y(0)=y_{0}$ (initial condition is given)

Step 2:

Set $t_{i}=i h$, with $t_{0}=0$ and $t_{k}=1, i=0,1, \ldots, k$.

\section{Step 3:}

for $i=1,2, \ldots, k-1$

$$
Y^{\prime}\left(t_{i}\right) \approx \frac{Y\left(t_{i+1}\right)-Y\left(t_{i-1}\right)}{2 h}
$$

for $i=k$

$$
Y^{\prime}\left(t_{i}\right) \approx \frac{3 Y\left(t_{i}\right)-4 Y\left(t_{i-1}\right)+Y\left(t_{i-2}\right)}{2 h}
$$

Use step 1 and step 2, 3 to find the approximate value of $y_{k}$. Where $h \approx \frac{1}{k}$ is interval length between nodes. 


\section{Numerical Examples}

In this section, we consider three integro-differential equations. We apply the system of equations in (3.8) and (3.9-3.10). The programs have been provided by MATLAB 7.8.

The $\mathbb{L}^{2}, \mathbb{L}^{\infty}$ error and rate of convergence are defined to be, respectively:

$$
\begin{aligned}
& e_{2}=\left\|y_{k}(t)-y_{e x}(t)\right\|_{2}=\left(\int_{0}^{1}\left(y_{k}(t)-y_{e x}(t)\right)^{2} d x\right)^{\frac{1}{2}} \\
& e_{\infty}=\max _{1 \leq i \leq 2 M}\left|y_{k}\left(t_{i}\right)-y_{e x}\left(t_{i}\right)\right| \\
& \rho_{2, \infty}=\frac{\log \left[e_{2, \infty}\left(\frac{k}{2}\right) / e_{2, \infty}(k)\right]}{\log (2)}
\end{aligned}
$$

where $y_{e x}(t)$ is the exact solution and $y_{k}(t)$ is the approximate solution obtained by Eq. (3.11-3.12).

4.1. Example. Consider the following linear Fredholm integro-differential equation:

$$
y^{\prime}(t)=\int_{0}^{1} e^{t s} y(s) d s+y(t)+\frac{1-e^{t+1}}{1+t},
$$

with initial condition $y(0)=1$.

The exact solution is as follows: $y(t)=e^{t}$.

The numerical results are shown in table (1) and in figures (1, 2). Table (1) shows the behaviour of the error for the norm $\mathbb{L}^{2}$ and norm $\mathbb{L}^{\infty}$ in function of the parameter of discretization $h$ for different values of $k$. Note that as $h$ approaches zero, the numerical solution converges to the analytical solution $y(t)$.

\begin{tabular}{||c||c||c||}
\hline \hline$k$ & $\mathbf{L}_{2}$ & $\mathbf{L}_{\infty}$ \\
\hline \hline 8 & $8.6353 e-002$ & $5.4116 e-002$ \\
\hline \hline 16 & $2.9590 e-002$ & $1.4152 e-002$ \\
\hline \hline 32 & $1.0405 e-002$ & $3.8126 e-003$ \\
\hline \hline 64 & $3.6778 e-003$ & $9.9140 e-004$ \\
\hline \hline 128 & $1.3009 e-003$ & $2.5290 e-004$ \\
\hline \hline 256 & $4.6012 e-004$ & $6.3875 e-005$ \\
\hline \hline 512 & $1.6271 e-004$ & $1.6051 e-005$ \\
\hline \hline 1024 & $5.7535 e-005$ & $4.0231 e-006$ \\
\hline \hline 2048 & $2.0343 e-005$ & $1.0071 e-006$ \\
\hline \hline 4096 & $7.1925 e-006$ & $2.5193 e-007$ \\
\hline \hline Convergence rate & 2.8284 & 3.9975 \\
\hline \hline
\end{tabular}

Table 1. The errors estimates $\mathbb{L}^{2}, \mathbb{L}^{\infty}$ and convergence rates $\rho_{2, \infty}$ 

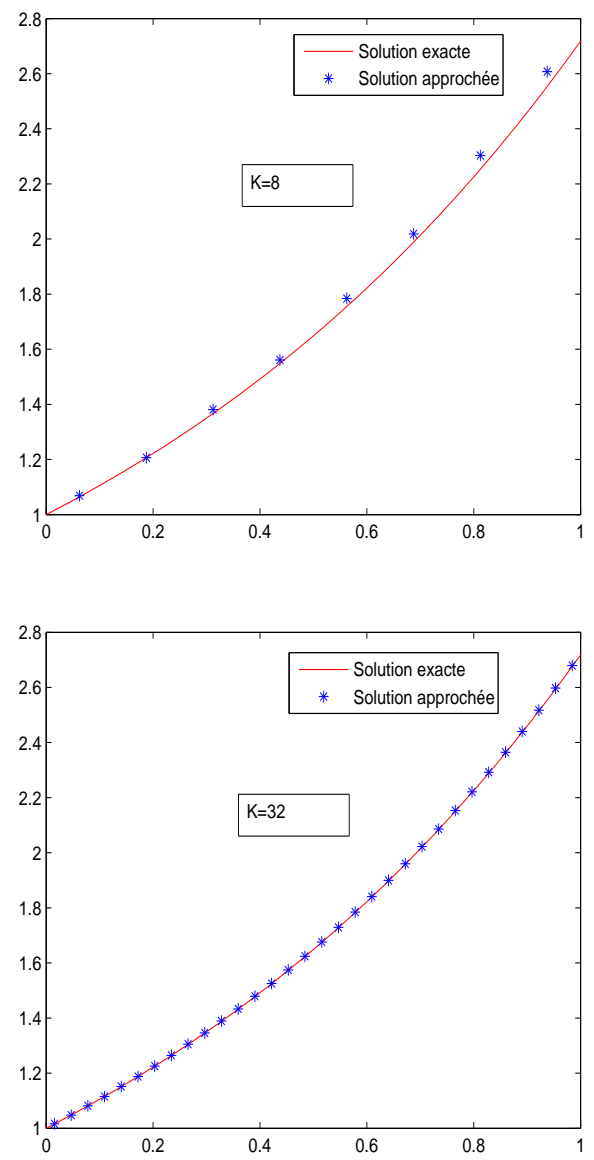

Figure 1. Comparison between approximate solution $y_{k}$ and exact solution $y_{e x}$ 


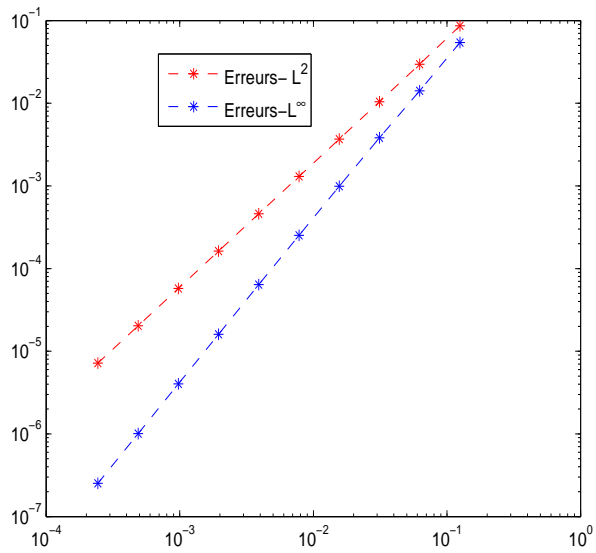

Figure 2. The errors $\mathbb{L}^{2}$ and $\mathbb{L}^{\infty}$ with different values of $k$.

We have also calculated the experimental rate of convergence $\rho_{2, \infty}$. We notice from Table (1) that the convergence rates are 2.8284 in $\mathbb{L}^{2}$ norm and 3.9975 in norm $\mathbb{L}^{\infty}$, which is approximately $2 \sqrt{2}$ and 4 respectively.

4.2. Example. Consider the following linear Fredholm integro-differential equation:

$$
y^{\prime}(t)=1-\frac{1}{3} t+\int_{0}^{1} t s y(s) d s
$$

with initial condition $y(0)=0$.

The exact solution is as follows: $y(t)=t$.

The numerical results are shown in table (2) and in figures $(3,4)$. Table (2) shows the behaviour of the error for the norm $\mathbb{L}^{2}$ and norm $\mathbb{L}^{\infty}$ in function of the parameter of discretization $h$ for different values of $k$. Note that as $h$ approaches zero, the numerical solution converges to the analytical solution $y(t)$.

\begin{tabular}{||c||c||c||}
\hline \hline$k$ & $\mathbf{L}_{2}$ & $\mathbf{L}_{\infty}$ \\
\hline \hline 8 & $9.2671 e-004$ & $6.4523 e-004$ \\
\hline \hline 16 & $4.7431 e-004$ & $2.1068 e-004$ \\
\hline \hline 32 & $1.1754 e-004$ & $4.5027 e-005$ \\
\hline \hline 64 & $4.1585 e-005$ & $1.1443 e-005$ \\
\hline \hline 128 & $1.4705 e-005$ & $2.8836 e-006$ \\
\hline \hline 256 & $5.1991 e-006$ & $7.2377 e-007$ \\
\hline \hline 512 & $1.8382 e-006$ & $1.8130 e-007$ \\
\hline \hline 1024 & $6.4990 e-007$ & $4.5369 e-008$ \\
\hline \hline 2048 & $2.2977 e-007$ & $1.1348 e-008$ \\
\hline \hline Convergence rate & 2.8284 & 3.9992 \\
\hline \hline
\end{tabular}

Table 2. The errors estimates $\mathbb{L}^{2}, \mathbb{L}^{\infty}$ and convergence rates $\rho_{2, \infty}$ 

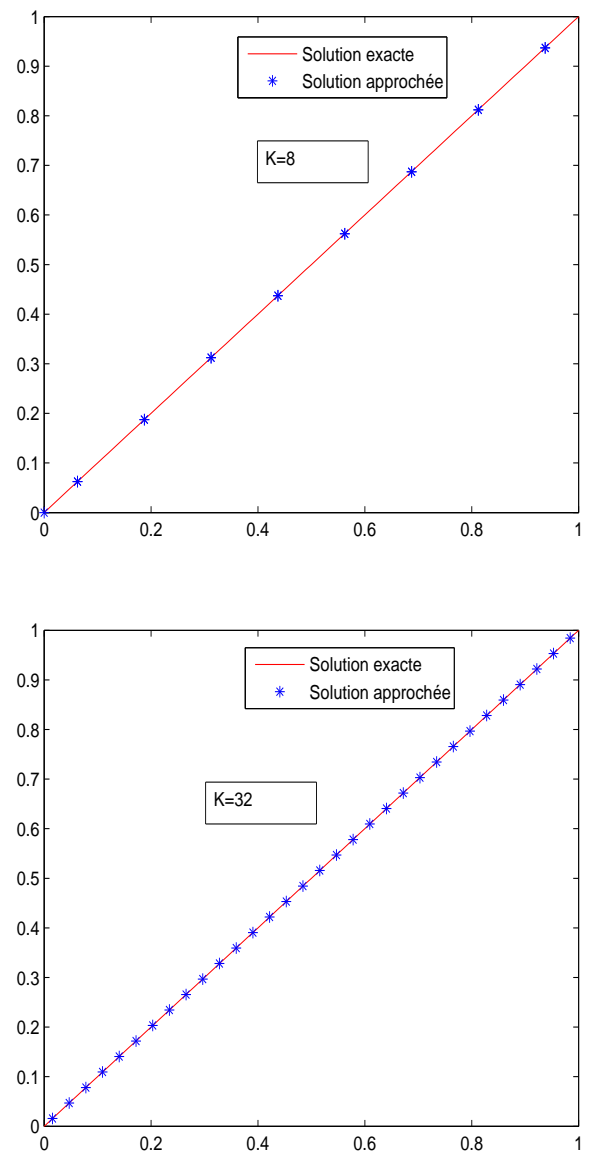

Figure 3. Comparison between approximate solution $y_{k}$ and exact solution $y_{e x}$ 


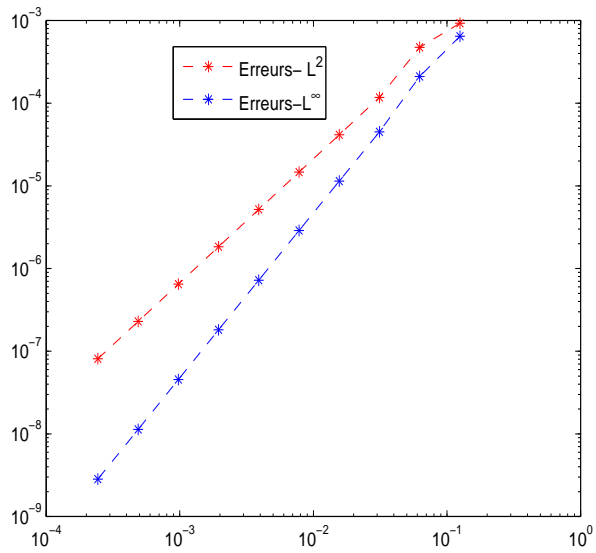

Figure 4. The errors $\mathbb{L}^{2}$ and $\mathbb{L}^{\infty}$ with different values of $k$.

We have also calculated the experimental rate of convergence $\rho_{2, \infty}$. We notice from Table (2) that the convergence rates are 2.8284 in $\mathbb{L}^{2}$ norm and 3.9975 in norm $\mathbb{L}^{\infty}$, which is approximately $2 \sqrt{2}$ and 4 respectively.

4.3. Example. Consider the following linear Fredholm integro-differential equation:

$$
(4.6 y)^{\prime}(t)=\int_{0}^{1} \sin (4 \pi t+2 \pi s) y(s) d s+y(t)-\cos (2 \pi t)-2 \pi \sin (2 \pi t)-\frac{1}{2} \sin (4 \pi x)
$$

with initial condition $y(0)=1$.

The exact solution is: $y(t)=\cos (2 \pi t)$. The numerical results are shown in Table $(3)$ and in figures $(5,6)$. Table (3) shows the behaviour of the error for the norm $\mathbb{L}^{2}$ and norm $\mathbb{L}^{\infty}$ in function of the parameter of discretization $h$ for different values of $k$. Note that as $h$ approaches zero, the numerical solution converges to the analytical solution $y(t)$.

\begin{tabular}{||c||c||c||}
\hline \hline$k$ & $\mathbf{L}_{2}$ & $\mathbf{L}_{\infty}$ \\
\hline \hline 8 & $4.9567 \mathrm{e}-001$ & $3.0091 \mathrm{e}-001$ \\
\hline \hline 16 & $1.7633 \mathrm{e}-001$ & $7.5816 \mathrm{e}-002$ \\
\hline \hline 32 & $6.2562 \mathrm{e}-002$ & $1.8990 \mathrm{e}-002$ \\
\hline \hline 64 & $2.2160 \mathrm{e}-002$ & $4.7500 \mathrm{e}-003$ \\
\hline \hline 128 & $7.8420 \mathrm{e}-003$ & $1.1877 \mathrm{e}-003$ \\
\hline \hline 256 & $2.7739 \mathrm{e}-003$ & $2.9696 \mathrm{e}-004$ \\
\hline \hline 512 & $9.8094 \mathrm{e}-004$ & $7.4244 \mathrm{e}-005$ \\
\hline \hline 1024 & $3.4686 \mathrm{e}-004$ & $1.8561 \mathrm{e}-005$ \\
\hline \hline 2048 & $1.2264 \mathrm{e}-004$ & $4.6402 \mathrm{e}-006$ \\
\hline \hline Convergence rate & 2.8284 & 3.9992 \\
\hline \hline
\end{tabular}

Table 3. The errors estimates $\mathbb{L}^{2}, \mathbb{L}^{\infty}$ and convergence rates $\rho_{2, \infty}$ 

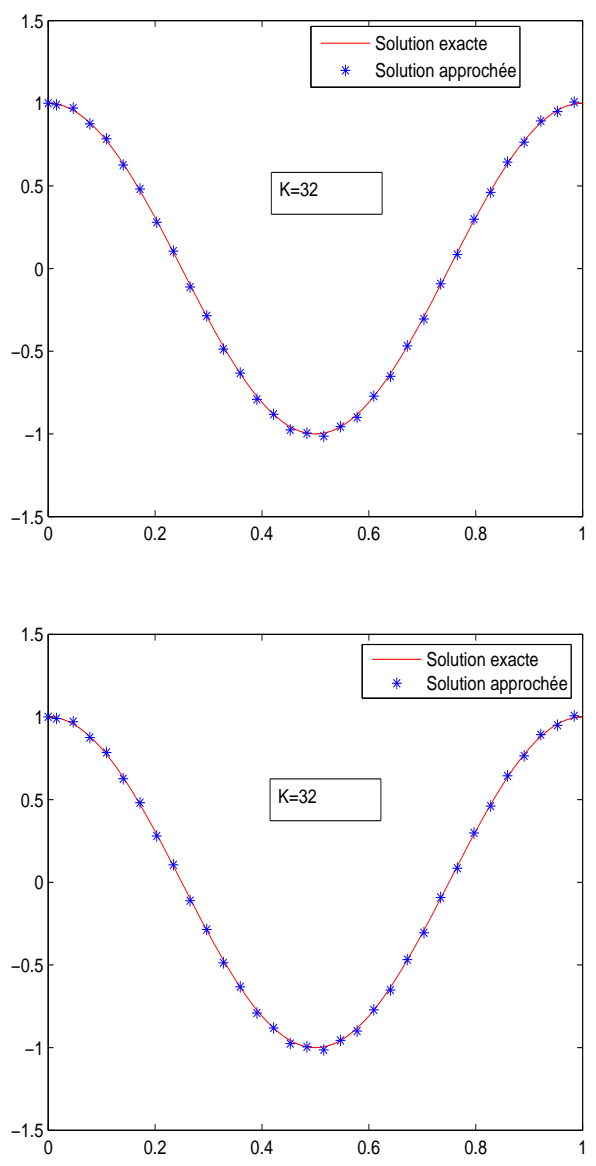

Figure 5. Comparison between approximate solution $y_{k}$ and exact solution $y_{e x}$ 


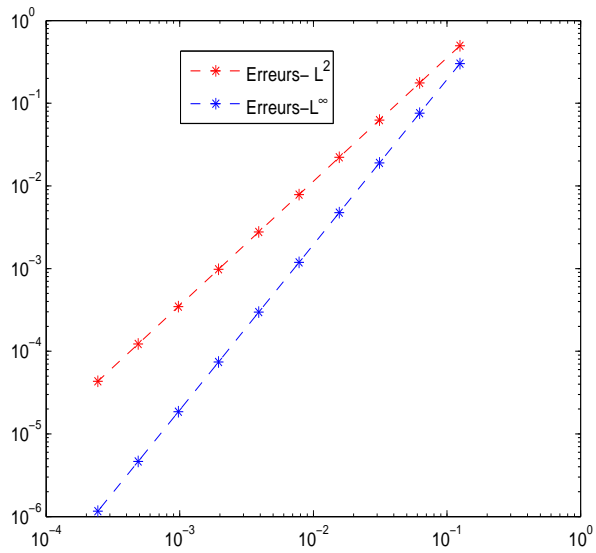

Figure 6. The errors $\mathbb{L}^{2}$ and $\mathbb{L}^{\infty}$ with different values of $k$.

We have also calculated the experimental rate of convergence $\rho_{2, \infty}$. We notice from Table (3) that the convergence rates are 2.8284 in $\mathbb{L}^{2}$ norm and 3.9975 in norm $\mathbb{L}^{\infty}$, which is approximately $2 \sqrt{2}$ and 4 respectively.

4.4. Example. Consider the Fredholm integral equation of the second kind:

$$
y^{\prime}(t)=\frac{1}{(\log 2)^{2}} \int_{0}^{1}\left(\frac{t}{1+s}\right) y(s) d s+y(t)-\frac{1}{2} t+\frac{1}{1+t}-\log (1+t)
$$

with initial condition: $y(0)=0$. The exact solution is: $y(t)=\log (1+t)$. The numerical results are shown in Table (4) and in figures $(7,8)$. Table (4) shows the behaviour of the error for the norm $\mathbb{L}^{2}$ and norm $\mathbb{L}^{\infty}$ in function of the parameter of discretization $h$ for different values of $k$. Note that as $h$ approaches zero, the numerical solution converges to the analytical solution $y(t)$.

\begin{tabular}{||c||c||c||}
\hline \hline$k$ & $\mathbf{L}_{2}$ & $\mathbf{L}_{\infty}$ \\
\hline \hline 8 & $1.0262 \mathrm{e}-002$ & $6.3139 \mathrm{e}-003$ \\
\hline \hline 16 & $4.0064 \mathrm{e}-003$ & $1.8118 \mathrm{e}-003$ \\
\hline \hline 32 & $1.4848 \mathrm{e}-003$ & $4.8413 \mathrm{e}-004$ \\
\hline \hline 64 & $5.3714 \mathrm{e}-004$ & $1.2506 \mathrm{e}-004$ \\
\hline \hline 128 & $1.9207 \mathrm{e}-004$ & $3.1777 \mathrm{e}-005$ \\
\hline \hline 256 & $6.8289 \mathrm{e}-005$ & $8.0088 \mathrm{e}-006$ \\
\hline \hline 512 & $2.4211 \mathrm{e}-005$ & $2.0103 \mathrm{e}-006$ \\
\hline \hline 1024 & $8.5720 \mathrm{e}-006$ & $5.0359 \mathrm{e}-007$ \\
\hline \hline 2048 & $3.0328 \mathrm{e}-006$ & $1.2602 \mathrm{e}-007$ \\
\hline \hline Convergence rate & 2.8284 & 3.9992 \\
\hline \hline
\end{tabular}

Table 4. The errors estimates $\mathbb{L}^{2}, \mathbb{L}^{\infty}$ and convergence rates $\rho_{2, \infty}$ 

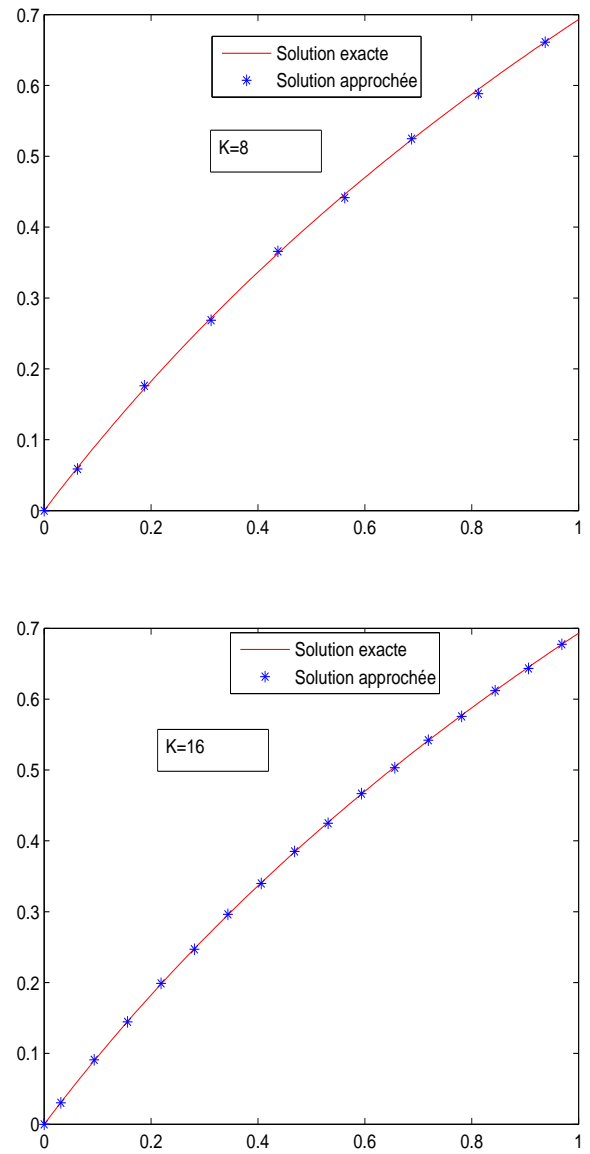

Figure 7. Comparison between approximate solution $y_{k}$ and exact solution $y_{e x}$ 


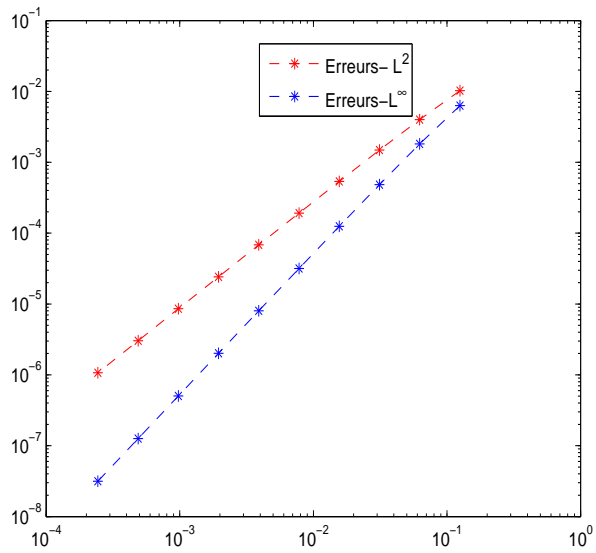

Figure 8. The errors $\mathbb{L}^{2}$ and $\mathbb{L}^{\infty}$ with different values of $k$.

We have also calculated the experimental rate of convergence $\rho_{2, \infty}$. We notice from Table (4) that the convergence rates are 2.8284 in $\mathbb{L}^{2}$ norm and 3.9975 in norm $\mathbb{L}^{\infty}$, which is approximately $2 \sqrt{2}$ and 4 respectively.

\section{CONCLUSION}

The proposed method is a powerful procedure for solving linear Fredholm integrodifferential. The examples analyzed illustrate the efficiency and reliability of the method presented and show that the method is very simple and effective. The obtained numerical solutions are very accurate, in comparison with the exact solutions. Results also indicate that the convergence rate is fast, and lower order approximations can achieve high accuracy.

ACKNOWLEDGMENT The authors gratefully acknowledge the comments made by anonymous referees.

\section{References}

[1] M.A. Abdou, Fredholm-Volterra integral equation with singular kernel, Appl. Math. Comput. 137 (2003), pp. 231-243.

[2] A. Ayad, Spline approximation for first Order fredholm delay integro-differential equations, International Journal of Computer Mathematics Volume 70, 1999-Issue 3.

[3] E. Babolian, New direct method to solve nonlinear Volterra-fredholm integral and Integrodifferential equations using Operational matrix with block-pulse Functions, Progress In Electromagnetics Research B, Vol. 8, 59-76, (2008).

[4] E. Babolian and Z. Masouri, Direct method to solve Volterra integral equation of the rst kind using operational matrix with block-pulse functions, J. Comput. Appl. Math.,220 (2008), 5157.

[5] S.BAZM, A direct rationalized haar functions method to Solve nonlinear two-dimensional fredholm Integral equations, Acta Universitatis Apulensis, No. 31 (2012), pp. 205-221.

[6] H. Brunner, Collocation method for Volterra integral and related functional equations, Cambridge Monograph on Applied and Computational Mathematics, Cambridge University Press, Cambridge, MA, (2004).

[7] C.F. Chen, C.H. Hsiao, Haar wavelet method for solving lumped and distributed-parameter systems, IEE Proc. Control Theory Appl. 144 (1997) 87-94. 
[8] W.S. Chen, and W. Lin, Galerkin trigonometric wavelet methods for the natural boundary integral equations. Appl. Math. Comput., 2005, 121, 7992.

[9] En-Bing Lin, Wavelet Based Methods for Numerical Solutions of Two Dimensional Integral Equations, Mathematica Aeterna, Vol. 4, (2014), no. 8, 839-853.

[10] M. A. Fariborzi Araghia, S. Daliri and M. Bahmanpour, Numerical Solution of IntegroDifferential Equation by using Chebyshev Wavelet Operational Matrix of Integration, International Journal of Mathematical Modelling and Computations Vol. 02, No. 02, (2012), 127-136.

[11] A. Filiz, Numerical Solution of Parabolic Volterra Integro-Differential Equations via Backward-Euler Scheme, American Journal of Computational and Applied Mathematics (2013), 3(6): 277-282.

[12] U. Lepik and E. Tamme, Solution of nonlinear Fredholm integral equations via the Haar wavelet method, Proc. Estonian Acad. Sci. Phys. Math., (2007), 56, 1, 17-27.

[13] U. Lepik, Haar wavelet method for nonlinear integro-differential equations, Applied Mathematics and Computation 176 (2006) 324-333.

[14] F. Mirzaee and S. F. Hoseini, Solving systems of linear Fredholm integro-differential equations with Fibonacci polynomials, Ain Shams Engineering Journal (2014) 5, 271-283.

[15] F. Mirzaee and S. F. Hoseini, Numerical solution of linear Fredholm integral equations system by rationalized Haar functions method, International Journal of Computer Mathematics Volume 80, 2003-Issue 11.

[16] V. Mishra, H. Kaur, and R.C. Mittal Haar Wavelet Algorithm For Solving Certain Differential, Integral And Integro-Differential Equations, Int. J. of Appl. Math and Mech. 8(6): 69-82,(2012).

[17] M. S. Muthuvalu and J. Sulaiman, Numerical Solutions of Linear Fredholm Integral Equations Using Half-Sweep Arithmetic Mean Method, International Journal of Applied Mathematics and Computation. Volume 3(1), pp 77-86, (2011).

[18] Y. Ordokhani and M. Razzaghi, Solution of nonlinear Volterra-Fredholm-Hammerstein integral equa- tions via a collocation method and rationalized Haar functions, Applied Mathematics Letters 21 (2008), 4-9.

[19] S. Pandit and M. Kumar Haar Wavelet Approach for Numerical Solution of Two Parameters Singu- larly Perturbed Boundary Value Problems, Appl. Math. Inf. Sci. 8, No. 6, 2965-2974 (2014).

[20] G.M. Phillips, Analysis of numerical iterative methods for solving integral and integrodifferential equations, Comput. J., 13 (1970), 297-300.

[21] B. Raftari, Numerical Solutions of the Linear Volterm Integro-differential Equations: Homotopy Perturbation Method and Finite Difference Method, World Applied Sciences Journa19 (Special Issue of Applied Math): 07-12, (2010).

[22] L. Rahmani, B. Rahimi and M. Mordad, Numerical Solution of Volterra-Fredholm IntegroDifferential Equation by Block Pulse Functions and Operational Matrices, Gen. Math. Notes, Vol. 4, No. 2, June (2011), pp. 37-48.

[23] M.H. Reihani and Z. Abadi, Rationalized Haar functions method for solving Fredholm and Volterra integral equations, Journal of Computational and Applied Mathematics 200 (2007) $12-20$.

[24] A. R. Vahidi and T. Damercheli, A Modiffied ADM for Solving Systems of Linear Fredholm Integral Equations of the Second Kind, Applied Mathematical Sciences, Vol. 6, (2012), no. $26,1267-1273$. 
